\title{
Wirtinger type inequalities via fractional integral operators
}

\author{
Serkan Asliyüce
}

\begin{abstract}
In this study, we shall present Wirtinger type inequality in the fractional case with conformable fractional operators.
\end{abstract}

Mathematics Subject Classification (2010): 26A33, 26Dxx, 35A23.

Keywords: Fractional derivative, fractional integral, Wirtinger inequality.

\section{Introduction}

Fractional derivation and integration are as old as ordinary derivation and integration. The history of fractional calculus date back to 1695 . In that time, L'Hospital asked to Leibniz "what would be the one-half derivative of $x$ ?" After this conversation, many mathematicians tried to give a coherent definition of fractional derivative and integral operators. By the beginning of 20th century, some definitions of fractional derivative are introduced called Riemann-Liouville, Caputo, and Grünwald-Letnikov derivatives and so on. Fractional derivatives and integrals are studied widely in different branches of sciences like engineering, physics etc. For more knowledge about the history and applications, we refer to $[7,9,20]$.

The definitions we considered above mostly use the integral forms to define the fractional derivative. Riemann-Liouville and Caputo fractional derivatives use the Riemann-Liouville fractional integral defined by

$$
J_{a}^{\alpha} f(x)=\frac{1}{\Gamma(\alpha)} \int_{a}^{x}(x-t)^{\alpha-1} f(t) d t, m-1<\alpha<m, \alpha \in \mathbb{R} .
$$

And so, Riemann-Liouville and Caputo fractional derivatives are defined as

$$
D_{a}^{\alpha} f(x)=D^{m} J_{a}^{m-\alpha} f(x),
$$

and

$$
{ }^{C} D_{a}^{\alpha} f(x)=J_{a}^{m-\alpha} D^{m} f(x),
$$


respectively, where $m=\lceil\alpha\rceil$, and in the right hand of the definitions operator $D^{m}$ represents the ordinary derivative order $m$.

Apart from the linearity property, Riemann-Liouville or any of other fractional derivatives do not satisfy all properties of ordinary derivative. For example, Caputo derivative does not satisfy well-known formula of the product of two functions

$$
D(f(t) g(t))=g(t) D f(t)+f(t) D g(t),
$$

and Riemann-Liouville derivative does not satisfy

$$
D[c]=0, c \text { is constant. }
$$

Because of this facts, recently some mathematicians gave their efforts to give new definitions for fractional derivatives. To handle these difficulties, in 2014 Khalil et al. [15] gave a new definition of fractional derivative as

$$
T_{\alpha}(f)(t)=\lim _{\varepsilon \rightarrow 0} \frac{f\left(t+\varepsilon t^{1-\alpha}\right)-f(t)}{\varepsilon} .
$$

This definition, called conformable fractional derivative, satisfies many properties of ordinary derivatives like product rule, chain rule etc.

Because of inequalities were often used in the theoretical and applied mathematics, mathematicians studied about their extensions, generalizations and discretizations, see $[2,3,12,17,18]$ and references cited therein. And in the last decade authors started to transfer those inequalities known in the classical settings into fractional settings, both continuous and discrete cases, to make contributions to the development of fractional calculus theory $[4,5,8,10,11,21]$.

In this paper, we shall give the fractional analogues of Wirtinger type inequalities given below:

Theorem 1.1 (Wirtinger's Inequality). For any function $y \in C^{1}[0,1]$ such that

$$
y(0)=y(1)=0,
$$

we have

$$
\int_{0}^{1}\left(y^{\prime}(t)\right)^{2} d t \geq \pi^{2} \int_{0}^{1} y^{2}(t) d t .
$$

Remark 1.2. Although Fourier series are used for the proof of Theorem 1.1, this proof also can be made with Schwarz inequality. Then, in the second case, we have inequality

$$
\int_{0}^{1}\left(y^{\prime}(t)\right)^{2} d t \geq \int_{0}^{1} y^{2}(t) d t,
$$

where condition $y(1)=0$ is not needed.

In 1975, Hinton and Lewis [14] gave a generalized Wirtinger type inequality using Schwarz inequality: 
Theorem 1.3. For any positive $M \in C^{1}([a, b])$ with $M^{\prime}(t) \neq 0$, and $y \in C^{1}([a, b])$ with $y(a)=y(b)=0$, we have

$$
\int_{a}^{b} \frac{M^{2}(t)}{\left|M^{\prime}(t)\right|}\left(y^{\prime}(t)\right)^{2} d t \geq \frac{1}{4} \int_{a}^{b}\left|M^{\prime}(t)\right| y^{2}(t) d t .
$$

In 1999, Pena [19] gave the discrete analogue of the inequality established by Hinton and Lewis:

Theorem 1.4. For a positive sequence $\left\{M_{n}\right\}_{0 \leq n \leq N+1}$ satisfying either $\triangle M>0$ or $\triangle M<0$ on $[0, N] \cap \mathbb{Z}$

$$
\sum_{n=0}^{N} \frac{M_{n} M_{n+1}}{\left|\triangle M_{n}\right|}\left(\triangle y_{n}\right)^{2} \geq \frac{1}{\psi_{J}} \sum_{n=0}^{N}\left|\triangle M_{n}\right| y_{n+1}^{2}
$$

holds for any sequence $\left\{y_{n}\right\}_{0 \leq n \leq N+1}$ with $y_{0}=y_{N+1}=0$, where

$$
\psi_{J}=\left(\sup _{0 \leq n \leq N} \frac{M_{n}}{M_{n+1}}\right)\left[1+\left(\sup _{0 \leq n \leq N} \frac{\left|\triangle M_{n}\right|}{\left|\triangle M_{n+1}\right|}\right)^{1 / 2}\right]^{2} .
$$

For more discussion about the Wirtinger inequality, see [13, 16, 22] and references cited therein.

\section{Preliminaries}

In this section, we give basic definitions and fundamental results for conformable fractional operators, so the paper is self-contained.

Definition 2.1. The conformable fractional derivative of a function $f:[0, \infty) \rightarrow \mathbb{R}$ of order $0 \leq \alpha \leq 1$ is defined by

$$
T_{\alpha}(f)(t)=\lim _{\varepsilon \rightarrow 0} \frac{f\left(t+\varepsilon t^{1-\alpha}\right)-f(t)}{\varepsilon}
$$

for all $t>0$.

We note that if the conformable fractional derivative of function $f$ of order $\alpha$ exists, we say $f$ is $\alpha$-differentiable.

Theorem 2.2. Let $\alpha \in(0,1]$ and functions $f$ and $g$ be $\alpha$-differentiable at point $t>0$. Then following properties are hold:

(i) $T_{\alpha}(a f+b g)(t)=a T_{\alpha}(f)(t)+b T_{\alpha}(g)(t)$, for all $a, b \in \mathbb{R}$.

(ii) $T_{\alpha}\left(t^{m}\right)=m t^{m-\alpha}$, for all $m \in \mathbb{R}$.

(iii) $T_{\alpha}(c)=0$, for all constant functions $f(t)=c$.

(iv) $T_{\alpha}(f g)(t)=g(t) T_{\alpha}(f)(t)+f(t) T_{\alpha}(g)(t)$.

(v) $T_{\alpha}\left(\frac{f}{g}\right)(t)=\frac{g(t) T_{\alpha}(f)(t)-f(t) T_{\alpha}(g)(t)}{(g(t))^{2}}$

(vi) If, in addition, $\mathrm{f}$ is differentiable, then $T_{\alpha}(f)(t)=t^{1-\alpha} \frac{d f}{d t}$. 
Now, we give conformable fractional derivative of some functions:

(1) $T_{\alpha}\left(t^{m}\right)=m t^{m-\alpha}$, for all $m \in \mathbb{R}$.

(2) $T_{\alpha}(1)=0$.

(3) $T_{\alpha}\left(e^{a t}\right)=a t^{1-\alpha} e^{a t}, a \in \mathbb{R}$.

(4) $T_{\alpha}\left(e^{\frac{1}{\alpha} t^{\alpha}}\right)=e^{\frac{1}{\alpha} t^{\alpha}}$.

(5) $T_{\alpha}(\sin a t)=a t^{1-\alpha} \cos a t, a \in \mathbb{R}$.

(6) $T_{\alpha}(\cos a t)=-a t^{1-\alpha} \sin a t, a \in \mathbb{R}$.

(7) $T_{\alpha}\left(\sin \frac{1}{\alpha} t^{\alpha}\right)=\cos \frac{1}{\alpha} t^{\alpha}$.

(8) $T_{\alpha}\left(\cos \frac{1}{\alpha} t^{\alpha}\right)=-\sin \frac{1}{\alpha} t^{\alpha}$.

Definition 2.3. The conformable fractional integral of a function $f:[0, \infty) \rightarrow \mathbb{R}$ of order $0 \leq \alpha \leq 1$ is defined by

$$
I_{\alpha}^{a}(f)(t)=I_{1}^{a}\left(t^{\alpha-1} f\right)(t)=\int_{a}^{t} \frac{f(s)}{t^{1-\alpha}} d s,
$$

where the integral is the usual Riemann improper integral, and $\alpha \in(0,1)$.

Theorem 2.4. $T_{\alpha} I_{\alpha}^{a}(f)(t)=f(t)$, for $t \geq a$, where $f$ is any continuous function in the domain of $I_{\alpha}$.

Example 2.5. For $a=0$ and $\alpha=1 / 2$, the conformable integral of function

$$
f(t)=\sqrt{t} \cos t
$$

is

$$
I_{1 / 2}^{0}(\sqrt{t} \cos t)=\int_{0}^{t} \cos s d s=\sin t .
$$

For more information and applications on conformable fractional operators, we refer to $[1,6,15,21]$ and papers cited therein.

\section{Wirtinger type inequalities}

In this section, we will state Wirtinger type inequalities using conformable fractional operators.

We start giving the fractional analogue of the inequality given in (1.1) .

Theorem 3.1. For any function $f \in C^{\alpha}([a, b])$ such that $f(a)=0$, we have

$$
\int_{a}^{b}\left|T_{\alpha} f(t)\right|^{2} d_{\alpha} t \geq \frac{\alpha^{2}}{\left(b^{\alpha}-a^{\alpha}\right)^{2}} \int_{a}^{b}|f(t)|^{2} d_{\alpha} t,
$$

where $C^{\alpha}$ represents the family of $\alpha$-differentiable functions, and $\int_{a}^{t} g(s) d_{\alpha} s$ denotes the conformable fractional integral. 
Proof. From [1], we know

$$
I_{\alpha}^{a} T_{\alpha} f(t)=f(t)-f(a) .
$$

Using the condition $f(a)=0$, we have $f(t)=I_{\alpha}^{a} T_{\alpha} f(t)$, so on

$$
|f(t)| \leq \int_{a}^{t}\left|T_{\alpha} f(s)\right| d_{\alpha} s
$$

Applying Schwarz inequality to the right side of (3.2), we find

$$
\begin{aligned}
|f(t)| & \leq\left(\int_{a}^{t} d_{\alpha} s\right)^{1 / 2}\left(\int_{a}^{t}\left|T_{\alpha} f(s)\right|^{2} d_{\alpha} s\right)^{1 / 2} \\
& =\left(\frac{t^{\alpha}-a^{\alpha}}{\alpha}\right)^{1 / 2}\left(\int_{a}^{t}\left|T_{\alpha} f(s)\right|^{2} d_{\alpha} s\right)^{1 / 2} \\
& \leq \frac{\left(b^{\alpha}-a^{\alpha}\right)^{1 / 2}}{\alpha^{1 / 2}}\left(\int_{a}^{b}\left|T_{\alpha} f(s)\right|^{2} d_{\alpha} s\right)^{1 / 2}
\end{aligned}
$$

After squaring the inequality (3.3) and taking its conformable integral from $a$ to $b$, the desired result is obtained.

Secondly, we state the fractional analogue of the inequality given in Theorem 1.3.

Theorem 3.2. For any positive function $M \in C^{\alpha}([a, b])$ satisfying either $T_{\alpha}[M(t)]>0$ or $T_{\alpha}[M(t)]<0$ on $[a, b]$, we have

$$
\int_{a}^{b} \frac{M^{2}(t)}{\left|T_{\alpha}[M(t)]\right|}\left(T_{\alpha}[y(t)]\right)^{2} d_{\alpha} t \geq \frac{1}{4} \int_{a}^{b}\left|T_{\alpha}[M(t)]\right| y^{2}(t) d_{\alpha} t
$$

for any function $y \in C^{\alpha}([a, b])$ with $y(a)=y(b)=0$.

Proof. Suppose that $T_{\alpha}[M(t)]>0$. Then we have

$$
\begin{gathered}
I_{1}=\int_{a}^{b} T_{\alpha}[M(t)] y^{2}(t) d_{\alpha} t=\left.M(t) y^{2}(t)\right|_{a} ^{b}-\int_{a}^{b} M(t) T_{\alpha}\left[y^{2}(t)\right] d_{\alpha} t \\
=M(b) y^{2}(b)-M(a) y^{2}(a)-2 \int_{a}^{b} M(t) y(t) T_{\alpha}[y(t)] d_{\alpha} t=-2 \int_{a}^{b} M(t) y(t) T_{\alpha}[y(t)] d_{\alpha} t \\
\leq 2 \int_{a}^{b} M(t)|y(t)|\left|T_{\alpha}[y(t)]\right| d_{\alpha} t=2 \int_{a}^{b} \sqrt{\frac{M^{2}(t)}{T_{\alpha}[M(t)]}}\left|T_{\alpha}[y(t)]\right| \sqrt{T_{\alpha}[M(t)]}|y(t)| d_{\alpha} t .
\end{gathered}
$$


Using Schwarz inequality, we have

$$
\begin{aligned}
I_{1} & =\int_{a}^{b} T_{\alpha}[M(t)] y^{2}(t) d_{\alpha} t \\
& \leq 2\left(\int_{a}^{b} \frac{M^{2}(t)}{T_{\alpha}[M(t)]}\left(T_{\alpha}[y(t)]\right)^{2} d_{\alpha} t\right)^{1 / 2}\left(\int_{a}^{b} T_{\alpha}[M(t)] y^{2}(t) d_{\alpha} t\right)^{1 / 2} \\
& =2 \sqrt{I_{1} I_{2}}
\end{aligned}
$$

where

$$
I_{2}=\int_{a}^{b} \frac{M^{2}(t)}{T_{\alpha}[M(t)]}\left(T_{\alpha}[y(t)]\right)^{2} d_{\alpha} t .
$$

Dividing both sides of the above inequality by $\sqrt{I_{1}}$, we obtain

$$
\sqrt{I_{1}} \leq 2 \sqrt{I_{2}}
$$

Hence

$$
I_{2} \geq \frac{1}{4} I_{1}
$$

The proof is complete.

Remark 3.3. If we take $\alpha=1$ in (3.1), we have

$$
\int_{a}^{b}\left|f^{\prime}(t)\right|^{2} d t \geq \frac{1}{(b-a)^{2}} \int_{a}^{b}|f(t)|^{2} d t,
$$

$a=0, b=1$ with $\alpha=1$, we have

$$
\int_{0}^{1}\left|f^{\prime}(t)\right|^{2} d t \geq \int_{0}^{1}|f(t)|^{2} d t
$$

and this is the inequality given in (1.1).

Secondly, if we take $\alpha=1$ in (3.4), we have

$$
\int_{a}^{b} \frac{M^{2}(t)}{\left|M^{\prime}(t)\right|}\left(y^{\prime}(t)\right)^{2} d t \geq \frac{1}{4} \int_{a}^{b} M^{\prime}(t) y^{2}(t) d t,
$$

i.e., we have the inequality given in Theorem 1.3.

\section{References}

[1] Abdeljawad, T., On conformable fractional calculus, J. Comput. Appl. Math., 279(2015), 57-66.

[2] Agarwal, R.P., Difference Equations and Inequalities. Theory, Methods, and Applications, Marcel Dekker, 1992. 
[3] Agarwal, R.P., O’Regan, D., Saker, S., Dynamic Inequalities on Time Scales, Springer, 2014.

[4] Akin, E., Aslyüce, S., Güvenilir, A.F., Kaymakcalan, B., Discrete Grüss type inequality on fractional calculus, J. Inequal. Appl., (2015), 2015:174, 7 pp.

[5] Anastassiou, G.A., Multivariate fractional representation formula and Ostrowski type inequality, Sarajevo J. Math., 22(2014), no. 1, 27-35.

[6] Atangana, A., Baleanu, D., Alsaedi, A., New properties of conformable derivative, Open Math., 13(2015), 889-898.

[7] Baleanu, D., Diethelm, K., Scalas, E., Trujillo, J.J., Fractional Calculus. Models and Numerical Methods. Series on Complexity, Nonlinearity and Chaos, World Scientific Publishing, 2012.

[8] Budak, H., Sarikaya, M.Z., An inequality of Ostrowski-Grüss type for double integrals, Stud. Univ. Babeş-Bolyai Math., 62(2017), no. 2, 163-173.

[9] Diethelm, K., The Analysis of Fractional Differential Equations. An ApplicationOriented Exposition Using Differential Operators of Caputo Type, Springer-Verlag, 2010.

[10] Ferreira Rui, A.C., A discrete fractional Gronwall inequality, Proc. Amer. Math. Soc., 140(2012), no. 5, 1605-1612.

[11] Güvenilir, A.F., Kaymakçalan, B., Peterson, A.C., Taş, K., Nabla discrete fractional Grüss type inequality, J. Inequal. Appl., (2014), 2014:86, 9 pp.

[12] Hardy, G.H., Littlewood, J.E., Pólya, G., Inequalities, Cambridge University Press, 1988.

[13] Hilscher, R., A time scales version of a Wirtinger-type inequality and applications. Dynamic equations on time scales, J. Comput. Appl. Math., 141(2002), no. 1-2, 219-226.

[14] Hinton, D.B., Lewis, R.T., Discrete spectra criteria for singular differential operators with middle terms, Math. Proc. Cambridge Philos. Soc., 77(1975), 337-347.

[15] Khalil, R., Al Horani, M., Yousef, A., Sababheh, M., A new definition of fractional derivative, J. Comput. Appl. Math., 264(2014), 65-70.

[16] Liu, K., Fridman, E., Wirtinger's inequality and Lyapunov-based sampled-data stabilization, Automatica J. IFAC, 48(2012), no. 1, 102-108.

[17] Mitrinović, D.S., Pečarić, J.E., Fink, A.M., Classical and New Inequalities in Analysis, Kluwer Academic Publishers, 1993.

[18] Pachpatte, B.G., Mathematical Inequalities, Elsevier B.V., 2005.

[19] Pena, S., Discrete spectra criteria for singular difference operators, Math. Bohem., 124(1999), no. 1, 35-44.

[20] Podlubny, I., Fractional Differential Equations. An Introduction to Fractional Derivatives, Fractional Differential Equations, to Methods of Their Solution and Some of Their Applications, Academic Press, 1999.

[21] Sarikaya, M.Z., Gronwall type inequalities for conformable fractional integrals, Konuralp J. Math., 4(2016), no. 2, 217-222.

[22] Seuret, A., Gouaisbaut, F., Wirtinger-based integral inequality: application to time-delay systems, Automatica J. IFAC, 49(2013), no. 9, 2860-2866. 
Serkan Asliyüce

Amasya University

Faculty of Sciences and Arts

05100 İpekköy, Amasya, Turkey

e-mail: serkan.asliyuce@amasya.edu.tr, s.asliyuce@gmail.com 